\title{
The Collaborative Aging Research Using Technology Initiative: An Open, Sharable, Technology-Agnostic Platform for the Research Community
}

\author{
Zachary Beattie ${ }^{a, b, c}$ Lyndsey M. Miller ${ }^{a, d} \quad$ Carlos Almirola $^{e}$ \\ Wan-Tai M. Au-Yeunga, b, c Hannah Bernard ${ }^{a, b, c}$ Kevin E. Cosgrovea, b, c \\ Hiroko H. Dodge ${ }^{a}, b, c$ Charlene J. Gamboa ${ }^{f}$ Ona Golonka ${ }^{a, b}, c$
}

Sarah Gothard a, b, c Sam Harbison ${ }^{a, b}$, c Stephanie Irish ${ }^{a, b}, c$ Judith Kornfeld ${ }^{a}$, b, c Jonathan Lee ${ }^{a, b}, c$ Jennifer Marcoe ${ }^{a, b}, c \quad$ Nora C. Mattek ${ }^{a, b}, c \quad$ Charlie Quinn ${ }^{a, b}, c$ Christina Reynolds $a, b, c$ Thomas Riley ${ }^{a, b}, c \quad$ Nathaniel Rodrigues $a, b, c$ Nicole Sharma ${ }^{a}, b, c \quad$ Mary Alice Siqueland ${ }^{a}, b, c \quad$ Neil W. Thomas ${ }^{g}, h$

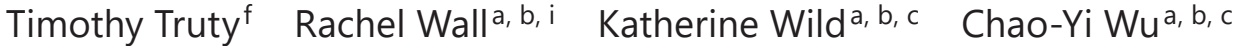
Jason Karlawish ${ }^{j} \quad$ Nina B. Silverbergk ${ }^{k}$ Lisa L. Barnes ${ }^{f} \quad$ Sara Czaja $^{e}$ I Lisa C. Silbert ${ }^{a}, b, c, i \quad$ Jeffrey Kaye ${ }^{a, b, c}$

a Oregon Center for Aging \& Technology, Oregon Health \& Science University, Portland, OR, USA; ${ }^{b}$ National Institute on Aging, Layton Aging \& Alzheimer's Disease Research Center, Oregon Health \& Science University, Portland, OR, USA; ' Department of Neurology, Oregon Health \& Science University, Portland, OR, USA; d School of Nursing, Oregon Health \& Science University, Portland, OR, USA; ${ }^{e}$ Center for Cognitive Neuroscience and Aging, Department of Psychiatry and Behavioral Sciences, University of Miami Miller School of Medicine, Miami, FL, USA; ${ }^{f}$ Rush Alzheimer's Disease Center, Rush University Medical Center, Chicago, IL, USA; ${ }^{9}$ Department of Medicine, University of

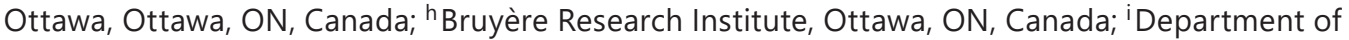
Neurology, Portland Veterans Affairs Medical Center, Portland, OR, USA; jDepartment of Medical

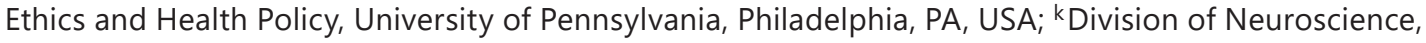
National Institute on Aging, National Institute of Health, Bethesda, MD, USA; 'Center on Aging and Behavioral Research, Division of Geriatrics and Palliative Medicine, Weil Cornell Medicine, New York, NY, USA

Keywords

Aging $\cdot$ Smart home $\cdot$ Pervasive computing $\cdot$ Digital biomarkers $\cdot$ Remote monitoring

\section{Abstract}

Introduction: Future digital health research hinges on methodologies to conduct remote clinical assessments and in-home monitoring. The Collaborative Aging Research Using Technology (CART) initiative was introduced to establish a digital technology research platform that could widely assess activity in the homes of diverse cohorts of older adults and detect 
meaningful change longitudinally. This paper reports on the built end-to-end design of the CART platform, its functionality, and the resulting research capabilities. Methods: CART platform development followed a principled design process aiming for scalability, use case flexibility, longevity, and data privacy protection while allowing sharability. The platform, comprising ambient technology, wearables, and other sensors, was deployed in participants' homes to provide continuous, long-term (months to years), and ecologically valid data. Data gathered from CART homes were sent securely to a research server for analysis and future data sharing. Results: The CART system was created, iteratively tested, and deployed to 232 homes representing four diverse cohorts (African American, Latinx, low-income, and predominantly rural-residing veterans) of older adults $(n=301)$ across the USA. Multiple measurements of wellness such as cognition (e.g., mean daily computer use time $=160-169 \mathrm{~min}$ ), physical mobility (e.g., mean daily transitions between rooms $=96-155$ ), sleep (e.g., mean nightly sleep duration $=6.3-7.4 \mathrm{~h}$ ), and level of social engagement (e.g., reports of overnight visitors $=15-45 \%$ ) were collected across cohorts. Conclusion: The CART initiative resulted in a minimally obtrusive digital health-enabled system that met the design principles while allowing for data capture over extended periods and can be widely used by the research community. The ability to monitor and manage health digitally within the homes of older adults is an important alternative to in-person assessments in many research contexts. Further advances will come with wider, shared use of the CART system in additional settings, within different disease contexts, and by diverse research teams.

(C) 2020 The Author(s)

Published by S. Karger AG, Basel

\section{Introduction}

The landscape of digital health is rapidly evolving. The ability to conduct remote clinical assessment and in-home monitoring has become an urgent public health priority due to the COVID-19 global pandemic [1,2], especially among older adults who are at greatest risk of COVID-19-related morbidity and mortality [3, 4]. While the need for remote clinical assessments has become more acute due to the COVID-19 pandemic, the knowledge deficits regarding how to best design, deploy, assess, deliver, analyze, and act on the resulting data from these methodologies have become equally pronounced and prevent the realization of the full value of remote assessment. The state of digital health science is now at a crossroads, with urgent needs for both forging ahead with remote clinical assessments of the aging population and for gaining evidence-based knowledge around how best to accomplish this on a large scale. This tension existed prior to the pandemic, but it has been amplified by recent events.

Many specific technologies and uses of remote monitoring have been developed to advance aging research [e.g., 5-8]. These advances have tended to benefit a relatively small community of specialty research; the broader gerontological research communities have not seen more widespread uptake of these methods, despite the demonstrated feasibility and potential of smart home and pervasive computing platforms to uniquely inform aging research and healthcare of older adults [9-15]. A number of barriers have prevented sensor-based and remote monitoring research from becoming ubiquitous: technical challenges, a lack of standards, team science requirements and lack of specialty expertise, narrow real-world use cases, proprietary algorithms and other access-limiting intellectual property rights, and costs. To address these obstacles, there has been a need for an open, scalable, and flexible infrastructure and a set of tools that allow the integration of data from a wide range of technologies (from wearables to passive sensors), informing multiple functions and domains of life (e.g., physical, cognitive, behavioral, social, psychological), and that is ecologically valid 
(i.e., collected in the home rather than a laboratory or clinical setting). Such an infrastructure would remove the well-documented obstacles that serve as barriers for researchers seeking to accommodate a diversity of technologies, data streams, and participants and allow unimpeded progress toward the maturation of digital health science and its practical dissemination.

In this context, the Collaborative Aging Research Using Technology (CART) initiative was introduced as an interagency (National Institutes of Health and Department of Veterans Affairs) resource-related multicomponent project (RFA-AG-16-021) proposed to develop and validate an infrastructure for digital health monitoring and intervention delivery that could be incorporated into the daily life of a diverse population of older adults. Building upon the previous work of the Oregon Center for Aging \& Technology (ORCATECH) [16-22], the purpose of the CART initiative was to facilitate successful aging-in-place research through an infrastructure that would enable health and activity changes to be recorded continuously and unobtrusively for months to years and that could accommodate a wide range of current and future technologies. The objective of this paper is to report on the built end-to-end design of the CART system, its functionality, and the resulting research capabilities. A future paper will describe the results of the ongoing longitudinal CART site-based demonstration project.

\section{Methods}

Design

The design of the CART system for studying aging, and aging-in-place, was guided at all stages by several principles. First and foremost was the need to simultaneously assess multiple domains of health and wellness that may be relevant to multiple disciplines, rather than an emphasis on a single domain (e.g., only sleep or mobility), methodology (e.g., mobile health), or disease context. Many schemas of health and wellness exist [23]. We focused on those that were found to be most frequently studied in the literature (physical, emotional/ psychological, intellectual/cognitive, and social) and that have a high impact on independence for aging well. In selecting technologies to incorporate into the CART platform, the focus was on minimally obtrusive or "ambient" technologies that would result in the least amount of disruption to the daily life of participants and yield the most ecologically valid data. The principled design of CART also emphasized remaining in a precompetitive environment and being technology-agnostic by choosing technologies for the widest array of use cases, rather than promoting a specific technology or designing a technology with an eye toward commercialization capabilities. Finally, the design of the CART system was driven by principles that would improve and sustain the initiative, including scalability to diverse environments and among diverse participants, being open and sharable while protecting data privacy, and the longevity of the system (e.g., the ability to monitor in the long term and seamlessly replace or incorporate additional technologies within the system as needed over time).

The development of the CART platform was fast-tracked by expanding upon an existing technology platform at ORCATECH that represents over a decade of experience using technology for aging and Alzheimer's disease and related dementias research [16-22]. The ORCATECH platform has been successfully implemented to make many basic observations in the aging and Alzheimer's disease and related dementias fields, such as developing algorithms and studying the relevance of unobtrusive measurement of walking speed [24, 25], sleep activity [26], time out of home [27], human mobility patterns within the home [28], computer use [18], medication-taking adherence [29], driving patterns [20], metadata from weekly online behavior [30], and changes in activity patterns during an epidemic quarantine [31].

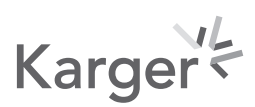


In developing the CART platform beyond the existing ORCATECH platform, the major methodological goals were (1) to improve sharability with other research groups (e.g., simplification and ease of use improvements, producing technology installation and maintenance guides), (2) to facilitate scalability from a few hundred homes to the capability of handling thousands of homes (e.g., moved from storing sensor data on one large MySQL database to a linearly scalable distributed NoSQL database), and (3) to achieve the flexibility to seamlessly integrate new technology (e.g., added an abstraction layer for communicating with sensors that utilize common wireless communication protocols). The choice and addition of technologies for the CART platform was an iterative process, driven by the above principles as well as technical specifications and availability. As improvements were made or new technology was added, they were first deployed to a small set of "beta" homes where they were tested for workability and effectiveness. Any issues found during the test period in the beta homes were corrected, and the new version was deployed back into the beta homes for re-testing. This iterative process was continued until no new bugs or issues were found with the improvements and/or new technology in the beta homes. After passing beta home testing, improvements and new technologies were deployed to all CART homes. The development team subsequently addressed technical issues that were found as the platform was deployed to the wider CART cohort, with proposed solutions iteratively tested as appropriate using the beta homes as described.

\section{Technology Back End}

The CART system (Fig. 1) featured a lambda architecture using open-source projects including Apache Cassandra, Apache Kafka, Apache Spark, and Google's Kubernetes to store data, manage pipelines, and run software in a distributed cluster computing environment. All software and data were stored and run on self-managed hardware in a secure private data center. However, the software was designed to be easily migrated to a cloud provider or run in a mixed environment if desired. The configurations of the cluster nodes were managed with the software management tool Puppet to ensure a homogeneous environment across the cluster. All external network traffic passed through a virtual private network (VPN) demilitarized zone that used access control lists to filter malicious packets. In addition to data collection needs, the software development process implemented continuous integration and deployment methodologies through the use of Gitlab and Kubernetes to manage software reviews, perform automated testing, and control software deployment to the field.

\section{Technology Front End}

The integration of sensor technology for collection of in-home data from the CART homes (i.e., the technology front end) used an abstraction layer that was designed to allow seamless integration of new sensor technologies that transmit data via standard wireless communication protocols (i.e., ZigBee, Bluetooth, and Wi-Fi) into the platform. While the implementation of the abstraction layer required significantly more upfront effort and development time than simply integrating known sensors directly into the CART platform, the abstraction layer limits future development effort and time by allowing new sensors to be added to the platform without necessitating changes to the technology back end (see Fig. 1 for a detailed depiction of the end-to-end CART system). A hub computer (Raspberry Pi 3 Model B) received and transferred all de-identified sensor data collected at the participant's home via a secure VPN connection to the secure research server. Daily rotation of the root user password, regular updates, and forwarding of all traffic through the VPN, among other measures, were implemented to ensure the security of the hub against known vulnerabilities. A penetration test and an external review of the security measures were also done to ensure the security of the hub. This hub computer broadcasted a wireless network in the participant's home, acted

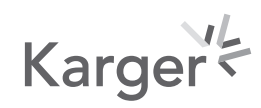




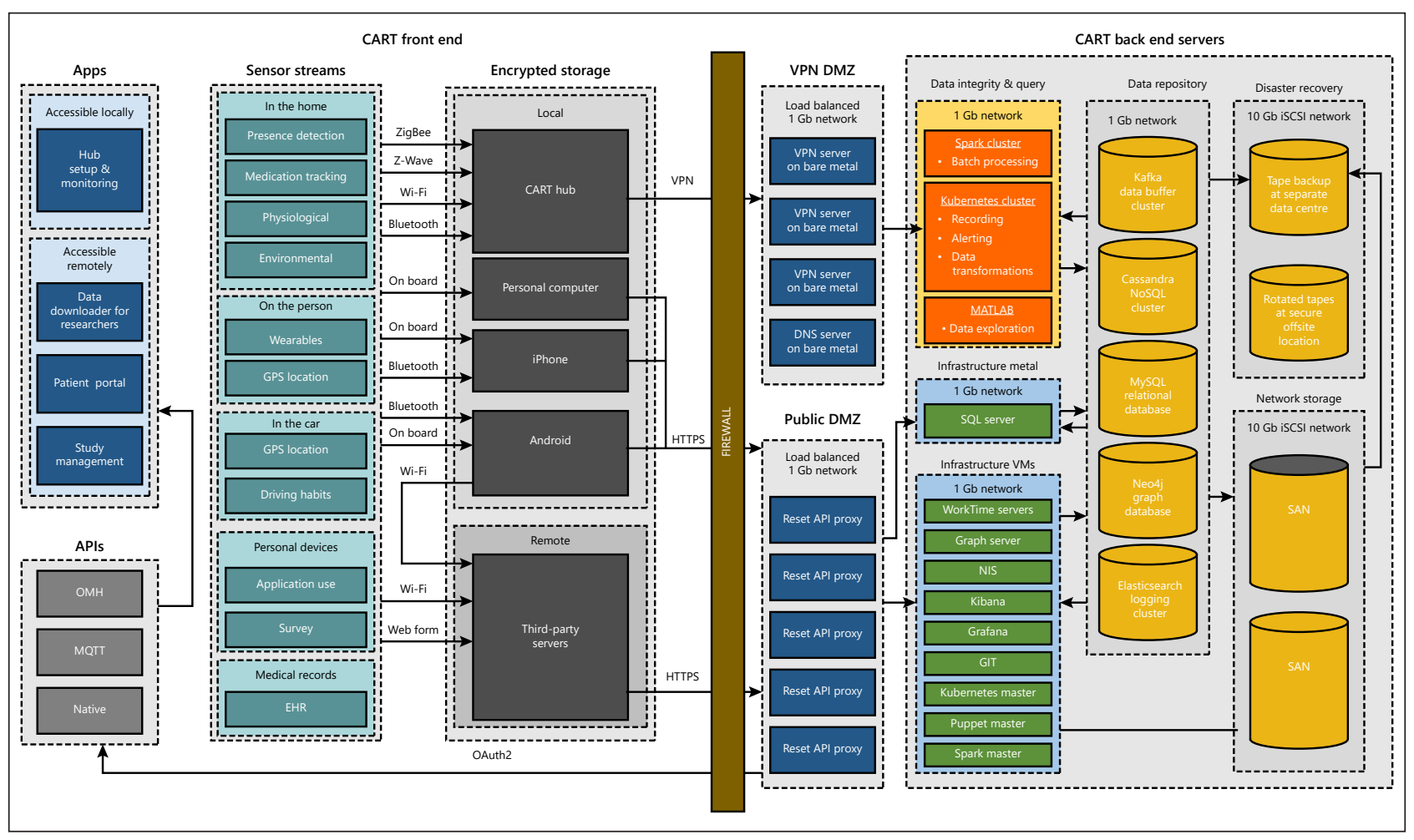

Fig. 1. The CART initiative's end-to-end system. The CART front end (shown on the left side of the "firewall") represents the sensors, data collection hub computers, and supporting applications that operate in participant homes. The CART back end (shown on the right side of the "firewall") represents how the data are stored and processed after they have been uploaded from the hub computers in participant homes. API, application programming interface; CART, Collaborative Aging Research Using Technology; DMZ, demilitarized zone; EHR, electronic health record; GPS, Global Positioning System; VPN, virtual private network.

as a client to a wireless or wired router, and checked in with the CART server to ensure that the in-home monitoring devices were up to date and properly identified. The in-home activity data were sent from the hub computer to the server on a continuous basis, and transmitted data were deleted from the device afterwards. Implementation of the front end technology into the CART platform is discussed herein based on the key functional areas of health that they were designed to measure (Fig. 2). However, it is important to note that most sensors have the potential to inform multiple domains of health.

While developing a robust technology platform was a major focus of the CART initiative, maintaining hundreds of homes with thousands of devices predictably involved addressing technical issues as they inevitably arose. Therefore, weekly reports for each home installed with the CART platform were generated that displayed the data integrity for each device in the home. Research technicians used these reports to generate technical issues. They would then address each technical issue either using the intuitive graphical interface (Fig. 3) to remotely access and fix the device that was not working properly or with a visit to the study participant's home if the device needed to be physically accessed to resolve the issue (e.g., a battery exchange). A technical help desk (reachable via phone, e-mail, or instant messaging) was also developed to offer assistance to research technicians on an as needed basis as they installed or maintained the CART platform technology. 


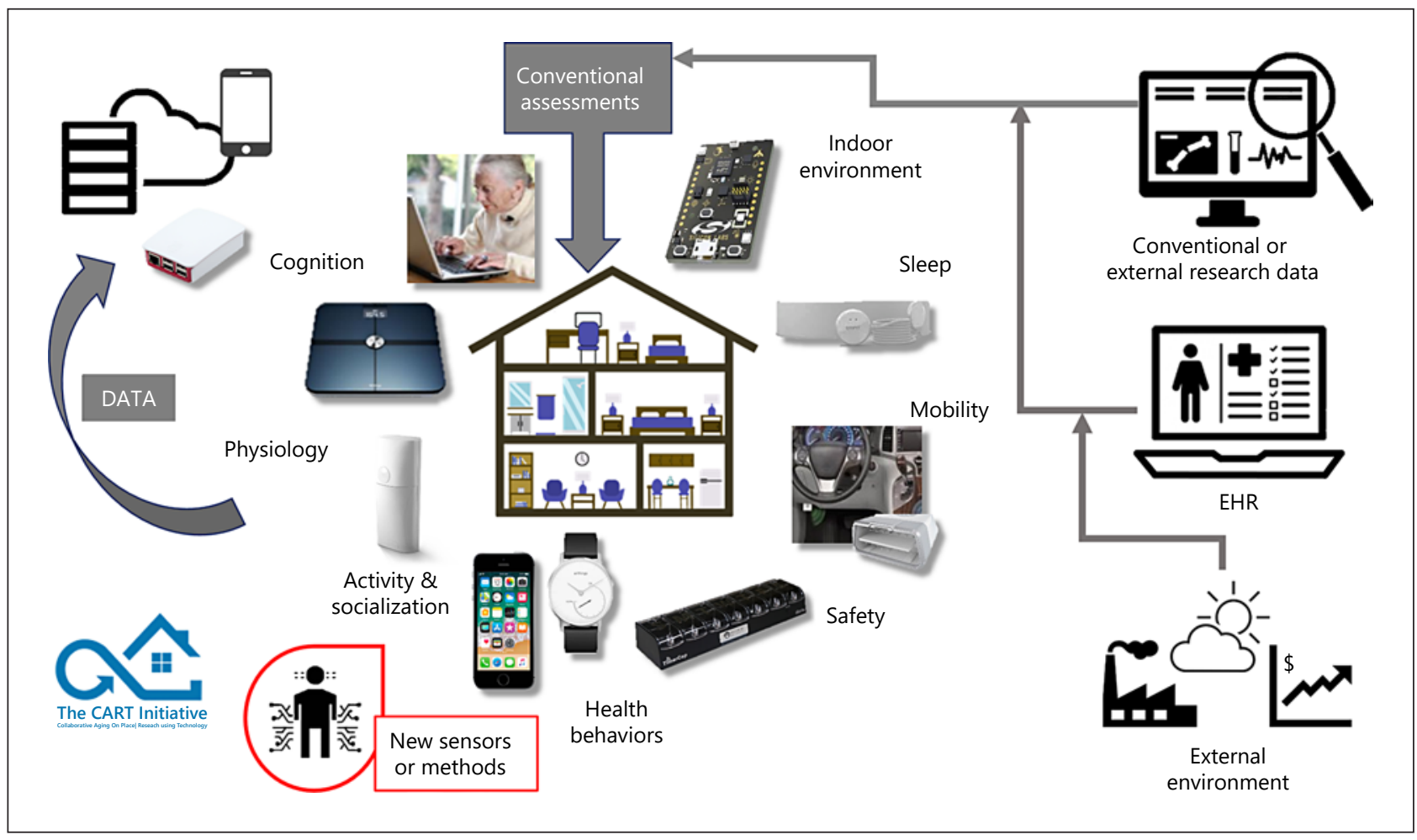

Fig. 2. Overview of sensors, devices, and data streams that can be integrated into the CART platform to monitor several health and wellness domains. CART, Collaborative Aging Research Using Technology; EHR, electronic health record.

\section{Domains of Health and Wellness Monitored}

Physical mobility in the CART platform was measured by two types of sensors. Passive infrared (PIR) sensors (NYCE, Burnaby, BC, Canada; i.e., motion activity detectors) monitored the amount of time participants spent in each room of their home and how often they moved around their home (e.g., transitions from room to room). These sensors were also used to measure participants' walking speed via a walking line of four PIR sensors placed at a carefully measured distance on the ceiling over a commonly used walking path in the home [17]. In addition, wearable devices (Withings Steel Watch; Withings, Issy-les-Moulineaux, France) [32] were used to monitor the activity level of CART participants. This actigraphy watch provided a long battery life (typically 8+ months) and had user-friendly features at a reasonable cost. In addition, the platform was designed and tested to incorporate other actigraphy devices (e.g., Actiwatch Spectrum, Phillips) for potential use in other researcher use cases, such as shorter term total activity measurements.

Sleep and nighttime behavior were monitored by the CART platform using a variety of devices and sensors. The PIR sensors detected inactive periods during the night when the participants were in their bedroom [21]. In addition, the Withings Steel wearable reported sleep duration and sleep activity using proprietary algorithms. Emfit (Finland) sleep mats were also incorporated into the platform after iterative testing in the beta homes. While also measuring sleep duration and sleep stages, the sleep mats collected a rich set of metrics surrounding participants' sleep, including, but not limited to, time in bed, time points when the participant left the bed, time points for restless periods during the night, and physiologic data (see below) [33]. 


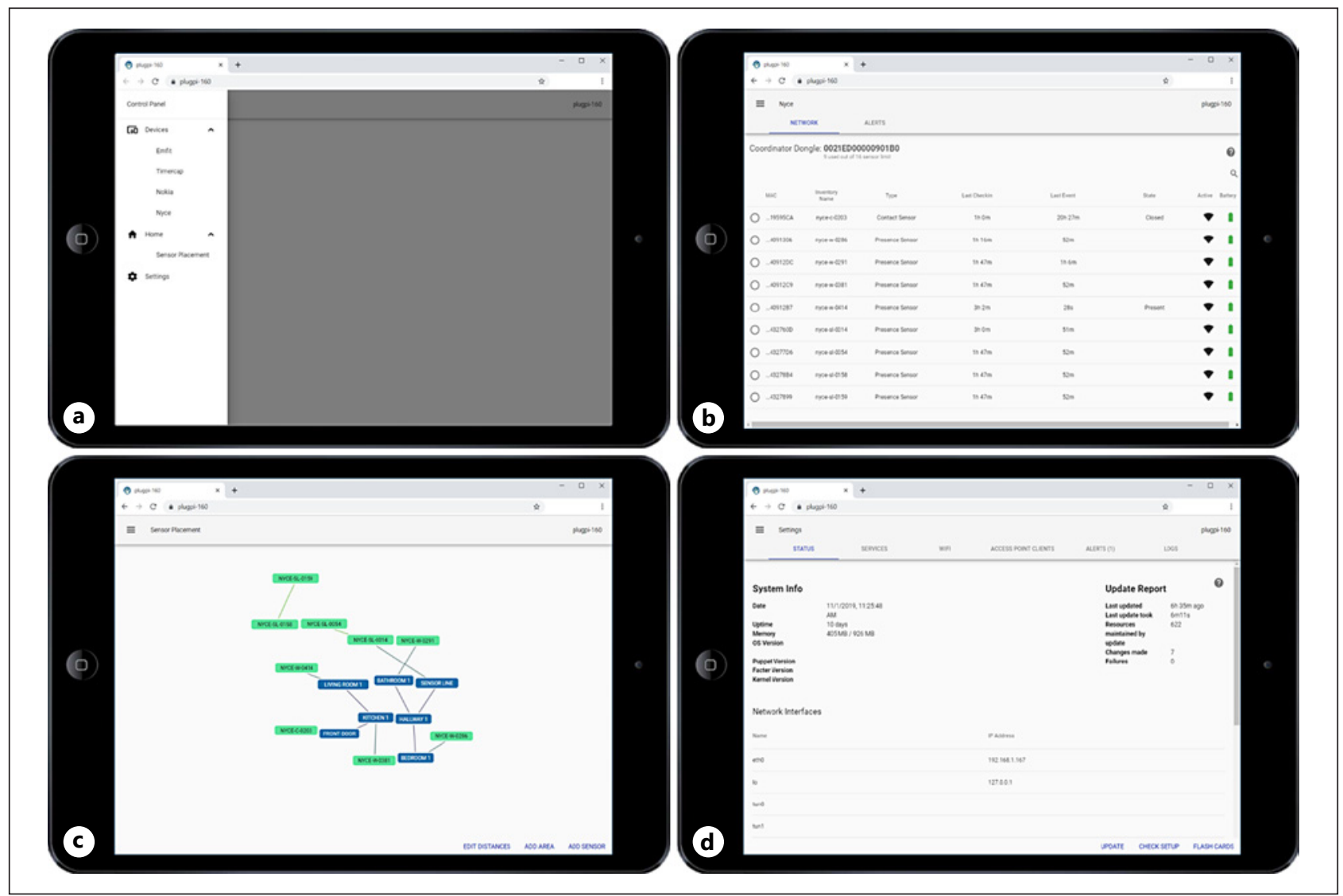

Fig. 3. Examples of the intuitive graphical interface used for platform installation and maintenance. a The main page acts as a portal to access information about the sensors and hub computer assigned to the participant home. $\mathbf{b}$ An example page for checking the status and connectivity of the PIR sensors (accessed under the "Devices" tab on the main page). c An example spider graph for setting and maintain where the PIR sensors are located (e.g., room) in the participant's home (accessed under the "Home" tab on the main page). d An example showing the status of the hub computer assigned to the participant's home (accessed under the "Settings" tab on the main page). PIR, passive infrared.

Physiologic function, including participants' weight and body mass composition (fat, muscle, and bone mass), were collected with the CART platform using the Withings Body Cardio digital scale [34]. The bioelectrical impedance analysis capability of the digital scale was disabled for safety reasons for participants with pacemakers. In such cases, the digital scale would only report participant weight. Evenly sampled nighttime heart and respiratory rates were collected by the Emfit sleep mats, in addition to heart rate variability.

Everyday cognition and safety were monitored through a number of sensors and metrics. Driving behavior was monitored with the CART platform using the Automatic Pro sensor (Automatic Labs, San Francisco, CA, USA) that was plugged into the OBD-II adapter of the participant's vehicle. Specific variables collected and processed from these sensors included, but are not limited to, the number of driving trips per day, the beginning and ending time for each trip, the duration spent driving on surface streets, the duration spent driving on highway roads, the number of hard braking events per trip, and the number of hard acceleration events per trip. In addition to driving behavior, the amount of time participants spent on their computers and which applications they used was collected using WorkTime software 
(WorkTime, Toronto, ON, Canada) software installed on their computers [35]. Medication taking (a prospective memory task) and adherence were measured using electronic pillboxes (TimerCap, Moorpark, CA, USA); these pillboxes recorded and wirelessly transmitted back to the hub computer information about when and which pillbox doors were opened and/or closed [36].

Social engagement was measured by the amount of time participants spent outside their homes each day, which was monitored from the PIR and contact door sensors. An algorithm made use of motion or lack of motion information from the PIR sensors and data from the contact sensors indicating when egress doors had been opened to predict when the participants were either present or not present in their home. Other measures of social interaction such as time together or apart (for couples) were also derived from the PIR sensor data. Information about overnight visitors and trips out of town were collected through a weekly online questionnaire described more fully below.

\section{Demonstration Cohort Sample and Conventional Clinical/Self-Reported Data Collection}

Four cohorts from community settings across the USA were recruited that represented a diverse population of older adults and environments. Included were low-income section 202 housing residents in Portland, African American and Latinx and Hispanic residents in Chicago and Miami, and veterans living in rural areas of Oregon and Washington. The African American cohort was part of the established Minority Aging Research Study [37] and as such also served as a demonstration of the ability to embed the system in an ongoing existing research program. Inclusion and exclusion criteria shared across CART sites included older adults age $\geq 62$ years living independently or with a partner (both partners provided written informed consent), current internet access or willingness to acquire access, basic computer use experience (send/receive e-mail), no dementia, and not being wheelchair-bound.

Standard conventional baseline and annual assessments obtained during in-person visits included a demographic form, personal and family medical histories and medication use, a physical and neuropsychiatric exam with a clinical assessor, questionnaires about activities of daily living and physical activity, measures of mood and affect (depressive symptoms, anxiety, loneliness, social isolation), and quality of life. Assessors entered subject clinical data into the web-based CART database housed in REDCap, an online data management and database creation tool $[38,39]$. The online supplementary Appendix (see www. karger.com/doi/10.1159/000512208) contains the full list of measures used and the methods for data collection in the CART initiative.

\section{Online Health and Activity Queries}

In addition to the baseline and annual clinical assessments, participants received a brief (13-item, $<5$ min to complete) weekly online health questionnaire about changes in behaviors or activities, including those that could affect in-home monitoring activity patterns (i.e., medication changes, falls, injuries, health changes, emergency room visits, depressive symptoms, pain experience, changes to living space, vacations, and visitors) and two annual surveys: a technology use survey and a subject annual survey. Meta-aspects of the completion of the weekly questionnaire (e.g., time to complete the survey, click counts) were recorded. The CART technology platform used survey capture information about subject technology access and experience, while the annual survey gathered self-reported sleep, loneliness, social isolation information, and quality of life. The weekly health update form was distributed from the Qualtrics Survey platform and sent through e-mail every Monday and, if it was not completed, a reminder e-mail was sent automatically on Wednesday. If the participant failed to complete the survey after the reminder e-mail, phone calls were made to improve data capture. Both annual surveys were automatically distributed from the Qualtrics Survey

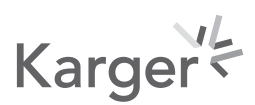


Table 1. Baseline participant characteristics by study site $(n=301)$

\begin{tabular}{lcccc}
\hline & OHSU & PVAMC & MIAMI & RUSH \\
\hline Number of participants & 77 & 125 & 31 & 68 \\
Number of households & 72 & 69 & 28 & 62 \\
Age, years & $71.5(6.5)$ & $70.9(6.3)$ & $73.7(7.2)$ & $76.6(5.9)$ \\
Female & $74 \%$ & $43 \%$ & $81 \%$ & $79 \%$ \\
Education, years & $15.2(2.6)$ & $14.5(2.1)$ & $15.1(2.5)$ & $16.2(2.9)$ \\
Race/ethnicity & & & & \\
$\quad$ White & $96 \%$ & $94 \%$ & $39 \%$ & $0 \%$ \\
$\quad$ Black & $0 \%$ & $<1 \%$ & $6 \%$ & $00 \%$ \\
$\quad$ Hispanic & $1 \%$ & $<1 \%$ & $0 \%$ & $0 \%$ \\
$\quad$ Other & $92 \%$ & $15 \%$ & $71 \%$ & $76 \%$ \\
Lives alone & $37 \%$ & $89 \%$ & $83 \%$ & $88 \%$ \\
Drives a car & $75 \%$ & $75 \%$ & $100 \%$ & $84 \%$ \\
Uses a smartphone & $24.7(3.0)$ & $23.3(3.4)$ & $24.7(3.5)$ & $\mathrm{N} / \mathrm{A}$ \\
MoCA & $\mathrm{N} / \mathrm{A}$ & $\mathrm{N} / \mathrm{A}$ & $\mathrm{N} / \mathrm{A}$ & $28.5(1.4)$ \\
MMSE & & $67.1(25.9)$ & $74.1(24.4)$ & $63.3(24.3)$ \\
SF-36 subscales & $62.2(25.5)$ & $76.0(23.3)$ & $80.2(24.1)$ & $87.2(17.2)$ \\
$\quad$ Physical function & $79.5(24.2)$ & $76.9(17.1)$ & $85.4(10.9)$ & $80.9(14.3)$ \\
Social function & $82.1(11.2)$ & & & $0 \%$ \\
Well-being & & & & \\
\hline
\end{tabular}

Values are presented as $n$, mean (standard deviation), or percentage. MIAMI, University of Miami; MMSE, Mini-Mental State Examination; MoCA, Montreal Cognitive Assessment; OHSU, Oregon Health \& Science University; PVAMC, Portland Veterans Affairs Medical Center; RUSH, Rush University Medical Center; SF-36, Short Form 36 Health Survey.

platform based on the previous year's completion date of those surveys. No reminders were sent, but CART coordinators contacted subjects who had not submitted responses to these surveys to ensure complete data collection.

Although not part of data collection from the four CART cohorts, the CART system was designed for researchers to be able to incorporate electronic health record (EHR) data as well as display CART home data to practitioners viewing standard EHRs. To facilitate the collection of EHRs, a user interface was built for participants to share their EHRs by logging into their EHR provider using the SMART authorization protocol by Fast Healthcare Interoperability Resources [40]. The tokens received by the EHR provider were then used by the server to pull, encrypt, and store the EHRs using the FHIR specification. The EHR could then be treated as another sensor stream within the CART system.

\section{Results}

During the 3.5-year CART initiative project period, the CART platform was created, iteratively tested, and ultimately deployed to 232 homes representing four diverse cohorts of older adults $(n=301)$ across the USA to collect and understand digital metrics of health, wellness, and preservation of independence. The baseline characteristics of the four cohorts can be found in Table 1 .

\section{Assessing Multiple Domains of Health and Wellness}

The resulting CART system monitored multiple health and wellness domains, including physical mobility, sleep and nighttime behavior, cognition, safety, and social engagement. The 
Table 2. Examples of unobtrusively captured activity data by study site $(n=301)$

\begin{tabular}{|c|c|c|c|c|c|c|}
\hline Domain & Activity metric & Device & OHSU $(n=77)$ & $\operatorname{PVAMC}(n=125)$ & MIAMI $(n=31)$ & RUSH $(n=68)$ \\
\hline \multirow{5}{*}{$\begin{array}{l}\text { Physical } \\
\text { mobility }\end{array}$} & mean daily total steps & watch & $3,154(2,445)$ & $2,984(2,523)$ & $2,470(1,651)$ & $1,949(1,325)$ \\
\hline & variability in daily total steps ${ }^{1}$ & watch & $1,505(929)$ & $1,435(1,170)$ & $1,300(738)$ & $1,106(691)$ \\
\hline & percent of days device worn & watch & $88(17)$ & $83(23)$ & $75(23)$ & $76(25)$ \\
\hline & mean room transitions ${ }^{2}$ & PIR sensors & $106(49)$ & $96(58)$ & $155(108)$ & $117(66)$ \\
\hline & variability in room transitions $^{1}$ & PIR sensors & $34(16)$ & $32(28)$ & $52(26)$ & $48(23)$ \\
\hline \multirow{3}{*}{$\begin{array}{l}\text { Sleep/ } \\
\text { nighttime } \\
\text { behavior }\end{array}$} & mean nightly sleep time, $\mathrm{h}$ & watch & $7.1(1.4)$ & $7.4(1.6)$ & $6.3(1.9)$ & $7.0(1.4)$ \\
\hline & variability in nightly sleep time ${ }^{1}$ & watch & $1.9(0.4)$ & $1.6(0.6)$ & $1.9(0.7)$ & $1.9(0.5)$ \\
\hline & percent of nights device worn & watch & $74(23)$ & $65(32)$ & $52(35)$ & $61(31)$ \\
\hline \multirow{5}{*}{$\begin{array}{l}\text { Physiologic } \\
\text { function }\end{array}$} & mean weight in pounds & scale & $193(47)$ & $200(44)$ & $159(30)$ & $176(35)$ \\
\hline & variability in weight $^{1}$ & scale & $3.5(5.0)$ & $2.7(3.6)$ & $1.8(1.2)$ & $5.1(8.0)$ \\
\hline & percent of days device used & scale & $52(34)$ & $47(35)$ & $50(36)$ & $50(30)$ \\
\hline & heart rate & sleep mat & $62.2(6.8)$ & $61.9(5.8)$ & $\mathrm{N} / \mathrm{A}$ & $66.7(6.9)$ \\
\hline & respiratory rate & sleep mat & $14.1(3.3)$ & $14.3(2.6)$ & $\mathrm{N} / \mathrm{A}$ & $14.7(2.8)$ \\
\hline \multirow{3}{*}{$\begin{array}{l}\text { Cognition/ } \\
\text { safety }\end{array}$} & daily total computer use, $\min ^{3}$ & WorkTime & $160(163)$ & $169(125)$ & $\mathrm{N} / \mathrm{A}$ & $\mathrm{N} / \mathrm{A}$ \\
\hline & percent of days computer used & WorkTime & $64(34)$ & 75 (27) & $\mathrm{N} / \mathrm{A}$ & $\mathrm{N} / \mathrm{A}$ \\
\hline & percent of days device used & MedTracker & $71(27)$ & $70(29)$ & $53(30)$ & $49(36)$ \\
\hline \multirow{5}{*}{$\begin{array}{l}\text { Social } \\
\text { engagement }\end{array}$} & online health form reports ${ }^{4}$ & & & & & \\
\hline & travel away overnight & QS & $41 \%$ & $57 \%$ & $65 \%$ & $48 \%$ \\
\hline & overnight visitors & QS & $15 \%$ & $45 \%$ & $31 \%$ & $44 \%$ \\
\hline & blue mood & QS & $18 \%$ & $14 \%$ & $8 \%$ & $13 \%$ \\
\hline & lonely & QS & $21 \%$ & $17 \%$ & $12 \%$ & $16 \%$ \\
\hline
\end{tabular}

Values are presented as mean (standard deviation) or percentage. MIAMI, University of Miami; OHSU, Oregon Health \& Science University; PIR, passive infrared; PVAMC, Portland Veterans Affairs Medical Center; QS, Qualtrics Survey; RUSH, Rush University Medical Center. ${ }^{1}$ Day-to-day variability is calculated as standard deviation of the 90 -day baseline period. ${ }^{2}$ Room transitions were only calculated for single-resident homes. ${ }^{3}$ Denominator $=$ days with computer use. ${ }^{4}$ Prevalence of ever reporting event during the 90 -day baseline period. Baseline: first 90 days of home monitoring.

focus on function over specific disease states or devices allowed the research platform to be relevant to multiple disciplines and to inform a wide array of potential outcomes of interest. Table 2 summarizes examples of sensor data for each of the health and wellness domains that were collected at baseline (averaged over the first 3 months following installation of the CART platform). The data acquired with the system were comparable to other data of representative older adults. For example, mean total sleep time was $7.1 \mathrm{~h}$ (range 6.3-7.4 h), which is similar to the $7 \mathrm{~h}$ per night of large population studies measured by self-report [41] and actigraphy [42]. The mean daily step count of the CART cohorts was 2,639 steps/day (range 1,9493,154 steps/day), which is similar to the normative steps per day for older adults previously reported by a national study [43]. Although some metrics generated by CART can be compared to prior studies, other metrics measured by CART, such as room transitions or time out of home, are novel and not yet outcome measures with wide experience. Figure 4 depicts examples of the breadth of derived data available for each CART participant.

\section{Use of Ambient Technologies}

Sensors and other technologies installed in participants' homes were chosen to be minimally obtrusive and ambient. Examples of how the sensors and technologies were installed in research participant homes are shown in Figure 5. This principle of the CART design increased the ecological validity of the data collected by the CART system and improved acceptability and user satisfaction. User-centered design concerns are typically lower when 


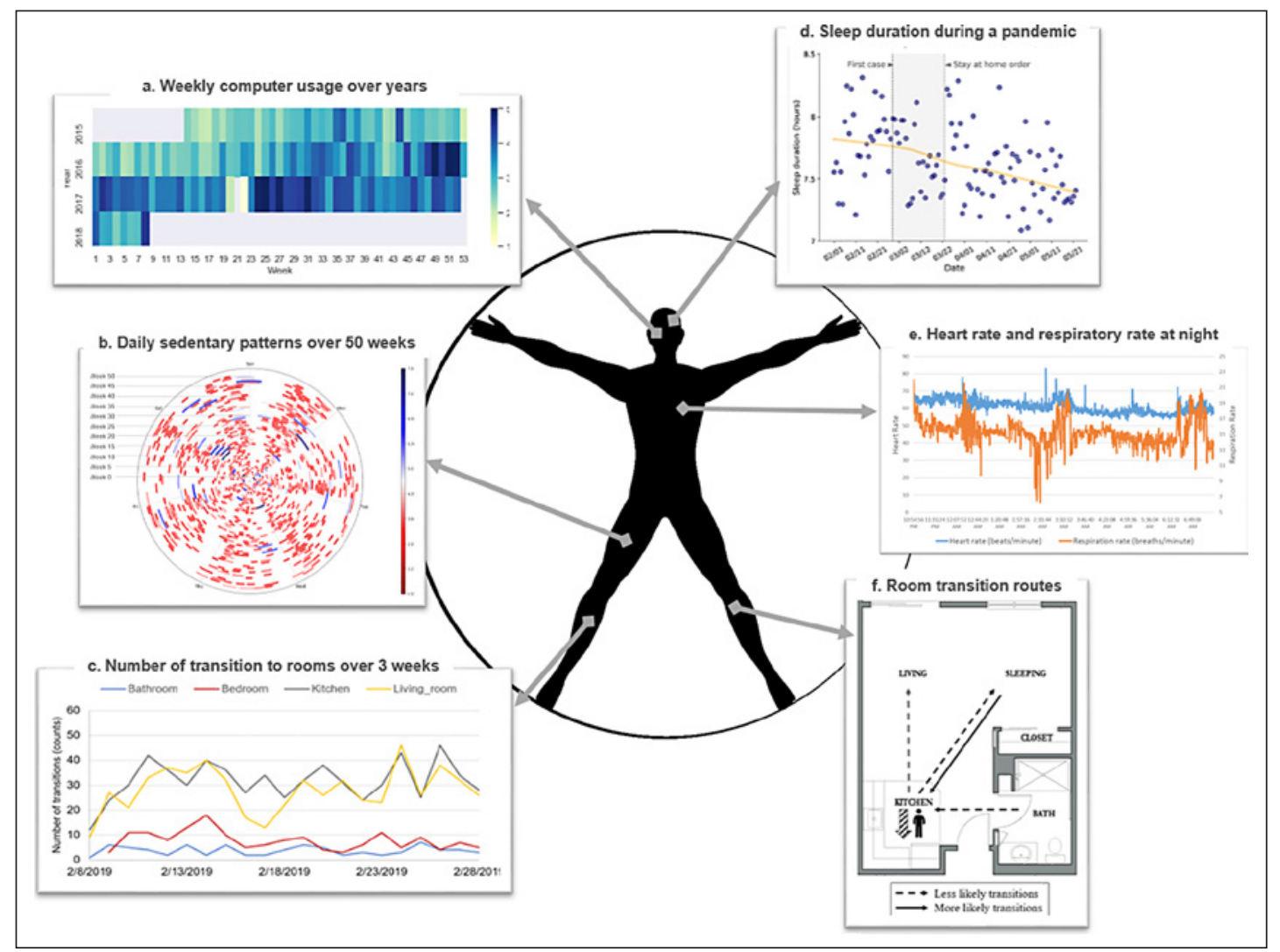

Fig. 4. Illustrations showing example sensor data for a CART participant. a Data illustration showing the weekly averaged daily computer use over 3 years from 2015 to 2018. Computer use was collected by the WorkTime software installed on the participant's computer. Darker blue suggests more computer use while lighter yellow suggests less computer use. The participant did not use their computer on week 22 of 2017. b Data illustration showing sedentary patterns in hours over a year. Sedentary time was calculated by the wearable device. Each circle represents a week, from Monday to Sunday. Blue lines suggest longer sedentary time while red lines suggest shorter sedentary time. $\mathbf{c}$ Data illustration showing the number of transitions to rooms (bathroom, bedroom, kitchen, living room) over 21 days. The number of transitions between rooms was calculated using the PIR motion sensors placed in each room. On average, this participant went to the kitchen and living room more and went to the bathroom least. $\mathbf{d}$ Data illustration showing sleep duration in hours during the COVID-19 pandemic from February 1 to May 21, 2020 in Oregon. Sleep duration was collected from the wearable device. The participant had a decline in sleep duration during the COVID-19 pandemic. The events of COVID-19 (first confirmed case, stay-at-home order) are highlighted in gray. e Data illustration showing variability of heart and respiratory rate for a night using data collected from the Emfit sleep mat. The participant had a drop in respiratory rate around 2:30 a.m. f Data illustration showing the probabilities of room transition routes over 1 month. Room transition routes (e.g., kitchen to living room) were detected using the PIR motion sensors in each room. The dashed lines indicate lower probability routes, while the solid line indicates higher probability routes that the participant would take over 1 month. CART, Collaborative Aging Research Using Technology; PIR, passive infrared.

there is no direct interaction with the technology; however, the informed consent process was carefully worded and reinforced to address the possibilities of "over-trust" (e.g., participants mistake sensors for an active monitoring system that can keep them safer) or "undertrust" (e.g., participants worry about privacy issues, such as video recording devices, which are not part of the platform) in the unobtrusive technologies. Questionnaires focusing on 


\section{Digital \\ Biomarkers}
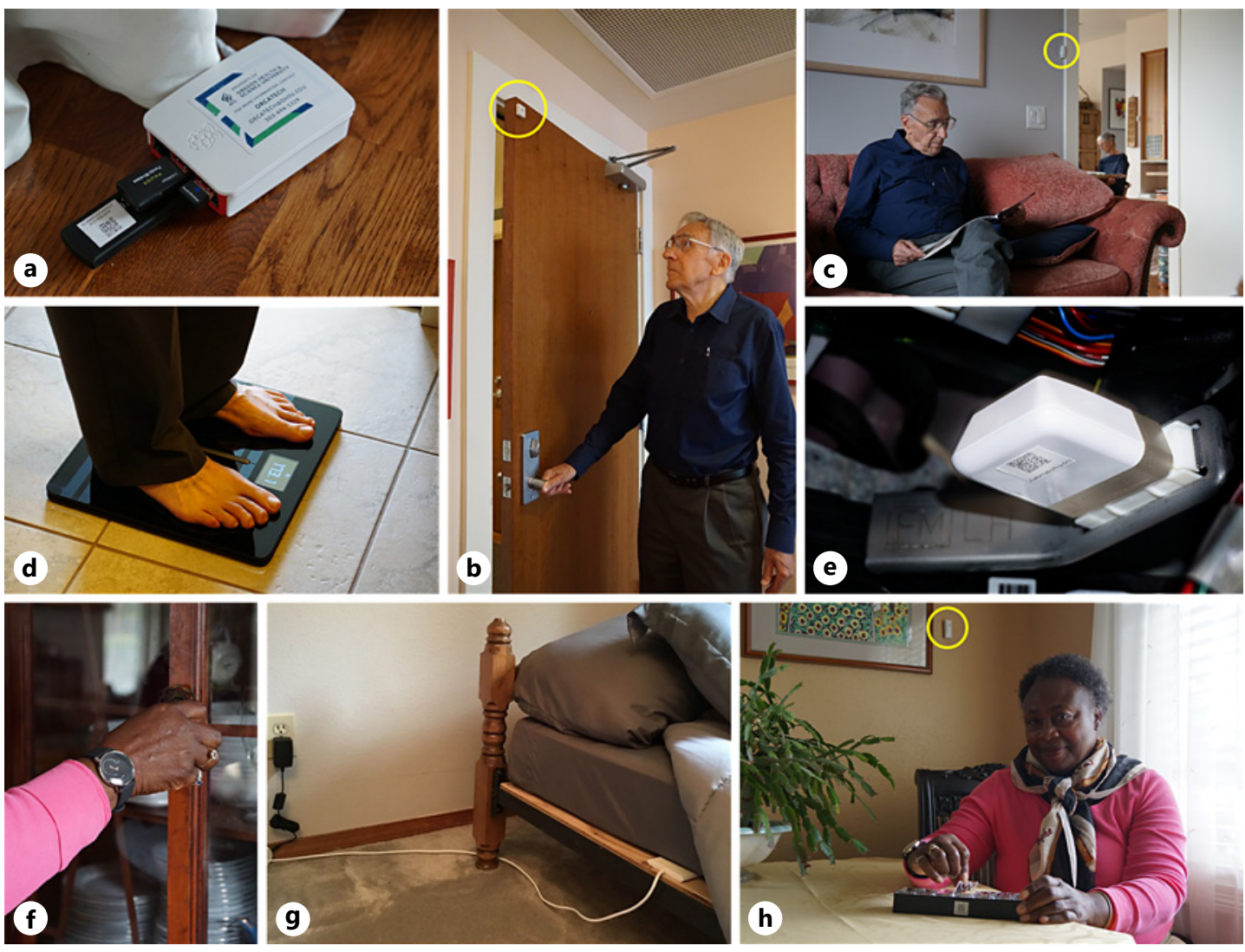

Fig. 5. Examples of how the sensors and technologies are installed in research participant homes. a A hub computer that is used to collect data from several of the sensors and securely transmit the data to the CART servers. b A contact door sensor (circled in yellow) that reports when the door is open and when the door is closed. c A PIR motion sensor (circled in yellow) that reports when an individual is in the room and when no one is present in the room. $\mathbf{d}$ A digital scale recording participant weight and body mass composition (fat, muscle, and bone mass). e A driving sensor plugged into the OBD-II adapter of a vehicle that records several metrics about driving such as, but not limited to, the number of driving trips per day, the beginning and ending time for each trip, the duration spent driving on surface streets, the duration spent driving on highway roads, the number of hard breaks per trip, and the number of hard accelerations per trip. $\mathbf{f}$ A consumer grade actigraphy watch that monitors the activity level and sleep of the wearer. $\mathbf{g}$ A sleep mat placed under a mattress that measures sleep duration and sleep stages amongst several other metrics about an individual's sleep. $\mathbf{h}$ Use of the electronic pillbox that records when the doors were opened and/or closed. As a note, a PIR motion sensor (circled in yellow) is also visible in this image. CART, Collaborative Aging Research Using Technology; PIR, passive infrared.

specific CART platform technologies (watch, pillbox, scale, computer use, vehicle sensor) assessed the usability of the technology. In general, technologies that required direct participant interaction (and thus had greater user-centered design issues) included the electronic pillbox ( $91 \%$ of CART participants reported taking some medications at baseline), the scale (daily use was required and readability of numbers was difficult for some), and the actigraphy watch, which required that participants wear it continuously on their nondominant wrist. Lastly, the weekly CART questionnaire required user interaction with their own computer, tablet, or smartphone device to complete. 


\section{Scalability}

The scalability of the CART system was demonstrated through the deployment to diverse environments (e.g., large rural homes and small urban low-income apartments) and to diverse cohorts of older adult participants (i.e., low-income section 202 housing residents, veterans, African Americans, and Latinx cohorts living in the Pacific Northwest, Chicago, and Miami areas of the USA). This was accomplished primarily through the creation of an intuitive graphical user interface (Fig. 3) that allowed the five CART technicians to deploy all sensors and devices without any technical coding skills (all five CART technicians held bachelor's degrees but had no previous experience in the role), such that the technician could be fully trained in a matter of days to deploy the entire study platform. Technicians were also provided with manuals that described in detail how to install and maintain the devices and study platform, thus increasing their capacity for independent problem-solving. Technicians reported that the usability of the platform was high in terms of ease of installation, had few safety concerns, and required little need for additional training ( $80 \%$ reporting that the CART platform was "easy to install" on the user questionnaire). When technicians did encounter a technical issue that fell outside of their scope of training, access to remote support was available; however, three-quarters of technician visits to homes for installation or maintenance required no remote support.

\section{Flexibility}

The CART initiative remained flexible by being agnostic to specific technologies used to monitor participants and by remaining in the precompetitive environment. When one type of sensor was not providing adequate data for a specific purpose, it was either replaced or augmented with the addition of another type of sensor. This flexibility was possible due to the abstraction layer and an iterative process of incorporating new technologies, which occurred by beta testing in the homes of six older adult participants who were selected based on the criteria of living in homes that were conveniently located for our technicians and having an expressed interest in the beta testing phase. For example, the Emfit sleep mat was introduced into the CART platform 20 months following deployment of the platform to the first home. The decision to incorporate the Emfit was made after discovering that approximately onethird of participants removed the actigraphy watch at night and thus the data on sleep were not being collected for this portion of the study sample.

\section{Open and Sharable while Protecting Data Privacy}

The CART system development yielded four possible avenues for retrieving information collected in the home. These options include: (1) an application programming interface that authenticates users with tokens for those who have programming experience and want to create tools using the system, (2) a graphical user interface that authenticates users with an OAuth2 protocol for viewing the data, (3) local access to real-time data streams from the hub computer over Wi-Fi with MQTT for users who want to be able to make informed decisions based on detected events, and (4) CART data can be requested by third-party researchers from the CART website (www.ohsu.edu/collaborative-aging-research-using-technology/ cart-data). To protect data privacy in transit, all information to and from the hub was encrypted over a VPN and application programming interface endpoints using the HTTPS protocol. Post hoc data acquisition was restricted by time and record to authenticated users using a realtime role base access control system which was maintained by a study coordinator. Additionally, sensor stream data were kept in a separate database from the protected health information data and only joined upon delivery to the authenticated user. Local access over Wi-Fi with MQTT contained only sensor data and required password-protected authentication and physical proximity to the study participant's home.

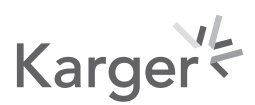




\section{Longevity of the System}

This report describes the developed system and "baseline" of the participant cohorts. To summarize the longevity or sustainability of the system, we report overall data capture experience once enrolled. A future report will detail the longitudinal follow-up of the CART cohorts. The current median number of days of sensor data collection is 615 days. Sensors and devices were specifically chosen for the longevity of their battery life (e.g., watch battery lasts 8+ months, NYCE batteries last up to $24+$ months) and their ability to send data wirelessly through the hub computer. The longevity of the CART system was further enhanced by the ability to seamlessly replace or incorporate additional technologies, as described above regarding the flexibility of the system.

\section{Discussion}

Over the course of 3.5 years, the CART initiative developed a minimally obtrusive digital health-enabled study system that monitored multiple domains of health and wellness, was scalable to diverse environments and participants, had the flexibility to incorporate different technologies as needed, provided data capture for extended periods of time, and could be widely used by the research community while still protecting participants' data privacy. The platform was deployed to four cohorts representing geographic, ethnic/racial, and socioeconomic diversity. In addition, the system was deployable within an existing ongoing research study (the Minority Aging Research Study at Rush University), demonstrating that the system could be employed and integrated into research studies that are already in operation with unique established protocols.

The use of digital or information-communication technologies in aging research has a history that extends decades. Rapid progression of the key technologies available has created a remarkably fertile field for harnessing these technologies for aging research with roots in classic telemedicine or telehealth [44]. In particular, the past decade has seen a transformation of this potential through increasing computing power, evolution of sensor capabilities and wireless communication protocols, growing internet connectivity often conceptualized as the Internet of Things, and data processing and analytic methodologies such as cloud computing and various artificial intelligence constructs $[13,45]$.

Despite this rapid evolution, or perhaps because of this high pace of development, this state of the art has yet to result in wide adoption of digital approaches in aging research beyond rather narrow focused use of various wearables or apps. Prior smart home or pervasive computing platforms for aging research have demonstrated the feasibility and potential of such platforms to uniquely inform research [9-15]. However, several barriers to their proliferation have been identified. The development of systems takes a multidisciplinary team that spans social, behavioral, and clinical science as well as disciplines among engineering, computer science, and data science. The process of creating effective systems requires realistic standardized approaches to the use cases, the users (patients as well as research teams), and the outcome measures. In a field that is moving fast, it is expensive and logistically challenging to assemble and maintain the teams necessary to create these systems, let alone to generate the clinical evidence that the data generated are valid, actionable, and ultimately not only able to demonstrate efficacy, but effectiveness. This is true whether in an academic or industry setting. Some home assessment platforms, as well as narrowly focused or one-off devices or apps, have found some penetration into the marketplace. Their proprietary nature, however, creates a drag on replication and iterative improvements without sharability of algorithms or the ability to rapidly modify a system to improve a feature. As a federally funded initiative, CART was conceived from its beginning as an open-source,

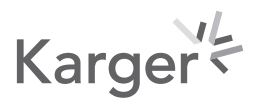


sharable system to spur digital research in aging. It thus attempts to improve upon these shortcomings.

Thus, the CART platform was developed to be flexible and purposefully designed without specific sensors or devices in mind. If a wearable is most appropriate for a particular study such as activity monitored through step counts, this can be accommodated. In other scenarios, fully passive sensors may be used such as to assess the activity of patients with late-stage dementia living in a memory care unit [46]. In yet other studies, a combination of strategies may be employed. As long as common standard communication protocols can be utilized to integrate a device or sensor, this new element can be propagated in a study. The current iteration of the CART platform utilizes ZigBee, Bluetooth, and Wi-Fi. However, by adding the appropriate antenna(s) to the hub computer, other wireless protocols could be implemented into the system. For example, Z-Wave, which is often used with Internet of Things devices, could be added to the platform, near-field communication could be utilized to securely read sensor/device tags near the hub computer, or the use of LoRaWAN could allow the communication between sensors and the hub computer to occur over longer distances. This allows for the platform to adapt with the constantly changing array of available sensing and computing technologies and needs of different researchers. This feature of CART also allows research groups to more rapidly design and test new technologies and new therapies for the field.

Making the CART platform accessible to the research community as a whole was a core principle of the CART initiative. A concerted effort has been made to keep the platform in the precompetitive environment to avoid ownership rights and exclusivity considerations that would limit the users of the CART platform and result in additional costs to users. In addition, the entire technology backend (Fig. 1) was designed to be completely sharable with research groups with the varying technical capabilities and to be run on an available cloud computing service if needed. For research groups without the desire or technical capabilities to host the platform themselves, standard operating practices were developed to guide collaborations such as extensive technology user manuals, site-visit training sessions, and the implementation of a staffed help desk. At the completion of the CART initiative, a total of 14 research groups spanning four countries and three continents were using the CART platform, indicating its widespread sharability beyond the four original CART initiative sites.

\section{Limitations}

A limiting factor to the future scalability of the CART system, and indeed most digital health platforms, is internet availability. In the current study, participants were required to have an internet connection in order to upload data from the hub computer to CART's servers and fill in weekly online surveys. However, it was found that several participants' internet connections were not reliable enough for the study protocol, typically due to remote locations of many in the rural veterans affairs cohort. For participants without reliable high-speed internet, we ended up providing a cellular internet connection. We also addressed the limiting nature of internet speed by ensuring robust caching on the hub computer's local database so that in the event that internet connection was lost, the data would be saved locally (i.e., on the hub computer) until they could be uploaded to the research server when a stable internet connection returned. Finally, we gated the amount of data that were being sent back and forth to the hub computer (frequency of check-ins and updates) to minimize the bandwidth needed to fit within cellular and slower connections.

Another technical limitation was the difficulty of monitoring the physical activity of people in multiperson residences. Although the CART system can monitor and disambiguate the activity within two-person homes, multigenerational dwellings that may include three or more people are more difficult. Person-specific activities such as medication taking or computer use may be less ambiguous to differentiate with simple solutions such as each participant

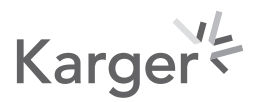


having their own electronic pillbox or using a computer profile (e.g., with a password, identity confirmation during sessions, or algorithmically by patterns of movement) [47].

Although the participants in this study were specifically recruited to represent four diverse cohorts of older adults across the USA, the average number of years of education reported by the resulting samples (averages ranged from 14.5 to 16.2 years) were higher than the national average, in which about half of adults ages $\geq 65$ years report at least some college education [48]. Future studies will need to include older adults with lower levels of education in order to demonstrate the feasibility of remote monitoring with this population. Finally, in the current pandemic climate, the ability of a technician to access participants' homes and complete installations is potentially a barrier. In order to respond to this barrier, future work will explore the feasibility of conducting remotely guided self-installations.

\section{Future Directions}

Eight out of 10 adults aged $\geq 65$ years in the USA are living with at least one chronic condition, such as heart disease, cancer, or Alzheimer's or a related neurodegenerative disease. Chronic diseases make up 90\% of the USD 3.5 trillion in annual healthcare costs, due in large part to frequent clinic visits and hospitalizations needed to manage symptoms and exacerbations [49]. There remains much to be learned about these disorders affecting a wide diversity of people. It is a priority to enable largely passive monitoring in order to study more populations which because of distance, disability, or complicated life situations has traditionally been challenging. An important future direction will be to apply the platform specifically to improve the longitudinal study of these chronic disease contexts and in particular take advantage of how high-dimensional digital data uniquely inform individual change. As such, the CART platform was developed to be open and sharable with the research community as a whole. The goal of the CART initiative was to develop a technology platform that researchers could use for these types of longitudinal studies without having to develop the technical data collection infrastructure themselves. At the time of publication, 14 groups were utilizing the CART platform, with research interests spanning from aging and Alzheimer's disease to cancer. Researchers interested in learning how to integrate the CART platform into their research can learn more about the platform and the CART initiative at www.carthome. org.

The need to facilitate the conduct of remote assessment has been heightened by the experience of the COVID-19 pandemic, where the ability to monitor and manage health digitally within the homes of older adults may be the safest way to assess patients and to provide care for older adults. While a complete analysis of CART participants' responses to the COVID-19 pandemic is beyond the scope of this paper, Figure $4 \mathrm{~d}$ is an example of decreased sleep duration observed in one CART participant during the pandemic, illustrating the utility of the data collected by the CART platform. Finally, the high-frequency, ecologically valid metrics generated by the digital assessment platform have an important role to play in measuring response to clinical trials, both pharmaceutical and behavioral [50]. They afford the opportunity to provide real-world outcomes with smaller sample sizes and/or shorter trial durations. Hence, future efforts will be directed at integrating the CART platform into clinical trials and treatment evaluations.

\section{Conclusions}

The CART initiative followed a principled design process to develop a platform that could monitor multiple domains of health and wellness, that was minimally obtrusive, and that was technology agnostic. The CART platform was designed to allow users to focus on the research

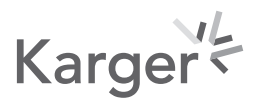


questions and not on the development of the technology needed to collect research data. The result was a scalable technology platform that can be widely used in many different use cases and environments with diverse populations by researchers with varied backgrounds.

\section{Statement of Ethics}

All subjects gave their written informed consent. The study protocol was approved by the Oregon Health \& Science Institutional Review Board (eIRB 17123), the Portland Veterans Affairs Institutional Review Board (IRB 4089), and the Rush University Institutional Review Board (16011407-IRB01). The University of Miami Review Board waived oversight to the Oregon Health \& Science Institutional Review Board (eIRB 17123).

\section{Conflict of Interest Statement}

The authors have no conflicts of interest to declare.

\section{Funding Sources}

This research was supported by the CART initiative through the Office of the Director, National Institutes of Health, the National Center For Advancing Translational Sciences, the National Institute of Biomedical Imaging and Bioengineering, the National Institute of Nursing Research, the National Institute on Aging, the National Institute of Neurological Disorders and Stroke, the National Cancer Institute, and the Veterans Administration, a National Institutes of Health grant (U2CAG0543701), a Veterans Administration grant (IIR 17-144), the Oregon Roybal Center for Translational Research on Aging (NIA P30AG024978), the Oregon Clinical Translational Research Institute CTSA award (UL1TR002369), and by funding from the National Institutes of Health (U2CAG054397, P30AG024978, P30AG008017, RF1AG22018, P30AG10161, and K01AG059839).

\section{Author Contributions}

Conception or design of the work: H.H. Dodge, N.B. Silverberg, L.L. Barnes, S. Czaja, L.C. Silbert, J. Kaye. Acquisition of data: Z. Beattie, C. Almirola, H. Bernard, K.E. Cosgrove, C.J. Gamboa, S. Gothard, S. Harbison, S. Irish, J. Lee, J. Marcoe, C. Quinn, T. Riley, N. Rodrigues, N. Sharma, M.A. Siqueland, T. Truty, R. Wall. Analysis of data: Z. Beattie, W.-T.M. Au-Yeung, H.H. Dodge, S. Gothard, N.C. Mattek, C. Reynolds, C.-Y. Wu. Interpretation of data: Z. Beattie, L.M. Miller, W.-T.M. Au-Yeung, H.H. Dodge, N.C. Mattek, K. Wild, C.-Y. Wu, J. Karlawish, L.L. Barnes, J. Kaye. Drafting of the manuscript: Z. Beattie, L.M. Miller, W.-T.M. Au-Yeung, H.H. Dodge, S. Gothard, N.C. Mattek, T. Riley, N. Rodrigues, C.-Y. Wu, L.L. Barnes, S. Czaja, L.C. Silbert, J. Kaye. Revision of the manuscript: Z. Beattie, L.M. Miller, W.-T.M. Au-Yeung, K.E. Cosgrove, H.H. Dodge, C.J. Gamboa, O. Golonka, S. Gothard, J. Kornfeld, J. Marcoe, N.C. Mattek, T. Riley, N. Sharma, N.W. Thomas, R. Wall, K. Wild, C.-Y. Wu, J. Karlawish, L.L. Barnes, S. Czaja, L.C. Silbert, J. Kaye. All authors approved the final version to be published and agree to be accountable for all aspects of the work in ensuring that questions related to the accuracy or integrity of any part of the work are appropriately investigated and resolved.

\section{Karger'k}




\section{References}

1 Dewar S, Lee PG, Suh TT, Min L. Uptake of Virtual Visits in A Geriatric Primary Care Clinic During the COVID-19 Pandemic. J Am Geriatr Soc. 2020 Jul;68(7):1392-4.

2 Farrell TW, Ferrante LE, Brown T, Francis L, Widera E, Rhodes R, et al. AGS Position Statement: Resource Allocation Strategies and Age-Related Considerations in the COVID-19 Era and Beyond. J Am Geriatr Soc. 2020 Jun; 68(6):1136-42.

3 Jordan RE, Adab P, Cheng KK. Covid-19: risk factors for severe disease and death. BMJ. 2020 Mar;368:m1198.

4 Richardson S, Hirsch JS, Narasimhan M, Crawford JM, McGinn T, Davidson KW, et al.; the Northwell COVID-19 Research Consortium. Presenting Characteristics, Comorbidities, and Outcomes Among 5700 Patients Hospitalized With COVID-19 in the New York City Area. JAMA. 2020 May;323(20):2052-9.

5 Skubic M, Guevara RD, Rantz M. Automated Health Alerts Using In-Home Sensor Data for Embedded Health Assessment. IEEE J Transl Eng Health Med. 2015 Apr;3:2700111.

6 Ghods A, Caffrey K, Lin B, Fraga K, Fritz R, Schmitter-Edgecombe M, et al. Iterative design of visual analytics for a clinician-in-the-loop smart home. IEEE J Biomed Health Inform. 2019 Jul;23(4):1742-8.

7 Kientz JA, Patel SN, Jones B, Price E, Mynatt ED, Abowd GD. The Georgia Tech aware home. CHI '08 Extended Abstracts on Human Factors in Computing Systems. Florence, Italy: Association for Computing Machinery; 2008. p. 3675-80.

8 Consel C, Dupuy L, Sauzéon H. HomeAssist: an assisted living platform for aging in place based on an interdisciplinary approach. Adv Hum Factors Ergon Healthc Med Devices. 2017;16:129-40.

9 Bakker JP, Goldsack JC, Clarke M, Coravos A, Geoghegan C, Godfrey A, et al. A systematic review of feasibility studies promoting the use of mobile technologies in clinical research. NPJ Digit Med. 2019 Jun 6;2:47.

10 Granja C, Janssen W, Johansen MA. Factors determining the success and failure of eHealth interventions: systematic review of the literature. J Med Internet Res. 2018 May;20(5):e10235.

11 Lussier M, Lavoie M, Giroux S, Consel C, Guay M, Macoir J, et al. Early Detection of Mild Cognitive Impairment With In-Home Monitoring Sensor Technologies Using Functional Measures: A Systematic Review. IEEE J Biomed Health Inform. 2019 Mar;23(2):838-47.

12 Mancioppi G, Fiorini L, Timpano Sportiello M, Cavallo F. Novel Technological Solutions for Assessment, Treatment, and Assistance in Mild Cognitive Impairment. Front Neuroinform. 2019 Aug;13(13):58.

13 Maresova P, Krejcar O, Barakovic S, Husic JB, Lameski P, Zdravevski E, et al. Health-related ICT solutions of smart environments for elderly: systematic Review. IEEE Access. 2020;17(8).

14 Piau A, Wild K, Mattek N, Kaye J. Current State of Digital Biomarker Technologies for Real-Life, Home-Based Monitoring of Cognitive Function for Mild Cognitive Impairment to Mild Alzheimer Disease and Implications for Clinical Care: systematic Review. J Med Internet Res. 2019 Aug;21(8):e12785.

15 Vegesna A, Tran M, Angelaccio M, Arcona S. Remote Patient Monitoring via Non-Invasive Digital Technologies: A Systematic Review. Telemed J E Health. 2017 Jan;23(1):3-17.

16 Kaye JA, Maxwell SA, Mattek N, Hayes TL, Dodge H, Pavel M, et al. Intelligent Systems For Assessing Aging Changes: home-based, unobtrusive, and continuous assessment of aging. J Gerontol B Psychol Sci Soc Sci. 2011 Jul;66(Suppl 1):i180-90.

17 Kaye J, Mattek N, Dodge H, Buracchio T, Austin D, Hagler S, et al. One walk a year to 1000 within a year: continuous in-home unobtrusive gait assessment of older adults. Gait Posture. 2012 Feb;35(2):197-202.

18 Kaye J, Mattek N, Dodge HH, Campbell I, Hayes T, Austin D, et al. Unobtrusive measurement of daily computer use to detect mild cognitive impairment. Alzheimers Dement. 2014 Jan;10(1):10-7.

19 Lyons BE, Austin D, Seelye A, Petersen J, Yeargers J, Riley T, et al. Pervasive Computing Technologies to Continuously Assess Alzheimer's Disease Progression and Intervention Efficacy. Front Aging Neurosci. 2015 Jun;7: 102.

20 Seelye A, Mattek N, Sharma N, Witter P, Brenner A, Wild K, et al. Passive assessment of routine driving with unobtrusive sensors: A new approach for identifying and monitoring functional level in normal aging and mild cognitive impairment. J Alzheimers Dis. 2017;59(4):1-11.

21 Hayes TL, Riley T, Pavel M, Kaye JA. Estimation of rest-activity patterns using motion sensors. Annu Int Conf IEEE Eng Med Biol Soc. 2010;2010:2147-50.

22 Kaye J, Reynolds C, Bowman M, Sharma N, Riley T, Golonka O, et al. Methodology for Establishing a CommunityWide Life Laboratory for Capturing Unobtrusive and Continuous Remote Activity and Health Data. J Vis Exp. 2018 Jul;(137):56942.

23 Miller G, Foster LT. Critical synthesis of wellness literature. Victoria, B.C.: University of Victoria; 2010.

24 Piau A, Mattek N, Crissey R, Beattie Z, Dodge H, Kaye J. When Will My Patient Fall? Sensor-Based In-Home Walking Speed Identifies Future Falls in Older Adults. J Gerontol A Biol Sci Med Sci. 2020 Apr;75(5):968-73.

25 Dodge HH, Mattek NC, Austin D, Hayes TL, Kaye JA. In-home walking speeds and variability trajectories associated with mild cognitive impairment. Neurology. 2012 Jun;78(24):1946-52.

26 Hayes TL, Riley T, Mattek N, Pavel M, Kaye JA. Sleep habits in mild cognitive impairment. Alzheimer Dis Assoc Disord. 2014 Apr-Jun;28(2):145-50.

27 Petersen J, Austin D, Mattek N, Kaye J. Time Out-of-Home and Cognitive, Physical, and Emotional Wellbeing of Older Adults: A Longitudinal Mixed Effects Model. PLoS One. 2015;10(10):e0139643.

28 Austin D, Cross RM, Hayes T, Kaye J. Regularity and predictability of human mobility in personal space. PLoS One. $2014 \mathrm{Feb}$;9(2):e90256. 
29 Austin J, Klein K, Mattek N, Kaye J. Variability in medication taking is associated with cognitive performance in nondemented older adults. Alzheimers Dement (Amst). 2017 Mar;6(1):210-3.

30 Seelye A, Mattek N, Sharma N, Riley T, Austin J, Wild K, et al. Weekly observations of online survey metadata obtained through home computer use allow for detection of changes in everyday cognition before transition to mild cognitive impairment. Alzheimers Dement. 2018 Feb;14(2):187-94.

31 Campbell IH, Austin D, Hayes TL, Pavel M, Riley T, Mattek N, et al. Measuring changes in activity patterns during a norovirus epidemic at a retirement community. Annu Int Conf IEEE Eng Med Biol Soc. 2011;2011: 6793-6.

32 Johnson KE, Alencar MK, Coakley KE, Swift DL, Cole NH, Mermier CM, et al. Telemedicine-based health coaching is effective for inducing weight loss and improving metabolic markers. Telemed J E Health. 2019 Feb;25(2): $85-92$.

33 Rauhala E, Virkkala J, Himanen SL. Periodic limb movement screening as an additional feature of Emfit sensor in sleep-disordered breathing studies. J Neurosci Methods. 2009 Mar;178(1):157-61.

34 Collier SR, McCraw C, Campany M, Lubkeman A, StClair P, Ji H, et al. Withings Body Cardio versus Gold Standards of Pulse-Wave Velocity and Body Composition. J Pers Med. 2020 Mar;10(1):17.

35 Austin J, Hollingshead K, Kaye J. Internet searches and their relationship to cognitive function in older adults: cross-sectional analysis. J Med Internet Res. 2017 Sep;19(9):e307.

36 Seelye A, Leese MI, Dorociak K, Bouranis N, Mattek N, Sharma N, et al. Feasibility of In-Home Sensor Monitoring to Detect Mild Cognitive Impairment in Aging Military Veterans: Prospective Observational Study. JMIR Form Res. 2020 Jun; 4(6):e16371.

37 Barnes LL, Shah RC, Aggarwal NT, Bennett DA, Schneider JA. The Minority Aging Research Study: ongoing efforts to obtain brain donation in African Americans without dementia. Curr Alzheimer Res. 2012 Jul; 9(6): 734-45.

38 Harris PA, Taylor R, Thielke R, Payne J, Gonzalez N, Conde JG. Research electronic data capture (REDCap) - a metadata-driven methodology and workflow process for providing translational research informatics support. J Biomed Inform. 2009 Apr;42(2):377-81.

39 Harris PA, Taylor R, Minor BL, Elliott V, Fernandez M, O’Neal L, et al.; REDCap Consortium. Building an international community of software platform partners. J Biomed Inform. 2019;95:103208.

40 Fast Healthcare Interoperability Resources. HL7 v4.0.1 2019. 2018. Available from: www.hl7.org/fhir/smartapp-launch/ [updated January 11, 2019].

41 Ohayon MM, Vecchierini MF. Normative sleep data, cognitive function and daily living activities in older adults in the community. Sleep. 2005;28(8):981-9.

42 Kurina LM, Thisted RA, Chen JH, McClintock MK, Waite LJ, Lauderdale DS. Actigraphic sleep characteristics among older Americans. Sleep Health. 2015 Dec;1(4):285-92.

43 Tudor-Locke C, Schuna JM Jr, Barreira TV, Mire EF, Broyles ST, Katzmarzyk PT, et al. Normative steps/day values for older adults: NHANES 2005-2006. J Gerontol A Biol Sci Med Sci. 2013 Nov;68(11):1426-32.

44 Bashshur RL, Shannon GW. History of Telemedicine: Evolution, Context, and Transformation. New Rochelle (NY): Mary Ann Liebert, Inc.; 2009.

45 Majumder S, Aghayi E, Noferesti M, Memarzadeh-Tehran H, Mondal T, Pang Z, et al. Smart homes for elderly healthcare - recent advances and research challenges. Sensors (Basel). 2017 Oct;17(11):E2496.

46 Au-Yeung WM, Miller L, Beattie Z, Dodge HH, Reynolds C, Vahia I, et al. Sensing a problem: proof of concept for characterizing and predicting agitation. Alzheimers Dement (N Y). 2020 Aug;6(1):e12079.

47 Seelye A, Hagler S, Mattek N, Howieson DB, Wild K, Dodge HH, et al. Computer mouse movement patterns: A potential marker of mild cognitive impairment. Alzheimers Dement (Amst). 2015 Dec;1(4):472-80.

48 United States Census Bureau. Educational attainment in the United States: 2019. Available from: www.census. gov/data/tables/2019/demo/educational-attainment/cps-detailed-tables.html.

49 Buttorff C, Ruder T, Bauman M. Multiple chronic conditions in the United States. Santa Monica, CA: Rand Corp.; 2017.

50 Thomas NW, Beattie Z, Marcoe J, Wright K, Sharma N, Mattek N, et al. An Ecologically Valid, Longitudinal, and Unbiased Assessment of Treatment Efficacy in Alzheimer Disease (the EVALUATE-AD Trial): Proof-of-Concept Study. JMIR Res Protoc. 2020 May;9(5):e17603. 